\title{
D'Amaurote à Dumocala : variations sur les rois en utopie
}

\section{L'utopie : une généalogie, une bibliothèque}

C'est à Rabelais qu'on attribue généralement la première utilisation en français du mot utopie que Thomas More avait forgé en 1516 avec la fortune que l'on sait ${ }^{1}$. Au chapitre 2 du Pantagruel, dans une énumération conforme à l'usage biblique de la généalogie, nous apprenons quels acteurs et quelles circonstances ont finalement présidé à la naissance du héros :

Gargantua, en son eage de quatre cens quatre-vingtz quarante et quatre ans, engendra son filz Pantagruel de sa femme nommée Badebec, fille du roy des Amaurotes en Utopie, laquelle mourut du mal d'enfant : car il estoit si merveilleusement grand et si lourd qu'il ne peut venir à lumière sans ainsi suffocquer sa mère. (Rabelais, 1973, p. 222)

Sous l'enflure du grotesque, une chose très sérieuse est dite : Gargantua, personnage énorme de fiction, est petit-fils du roi de l'Utopie originelle ; le livre de Rabelais est le descendant direct de celui de Thomas More.

Bien sûr, Rabelais ne s'est pas contenté de désigner l'utopie par son nom dans le premier de ses romans et cela jusqu'à six fois, en reprenant aussi le nom d'Amaurote que More avait donné à la ville capitale de l'Utopie. Sans toujours mobiliser explicitement le mot, il représentera encore la chose avec assez de précision en constituant,

- François Rosset - professeur de littérature française à l'Université de Lausanne. Adresse de correspondance : Chemin des Grives 2, CH-1024 Ecublens ; e-mail : Francois.Rosset@unil.ch

ORCID iD : https://orcid.org/0000-0002-3654-4320

1. T. More (1516). Libellus vere aureus, nec minus salutaris quam festivus, de optimo reipublicae statu deque nova insula Utopia. Louvain : Thierry Martens. 
dans le Gargantua, la fameuse abbaye de Thélème ; puis plus tard, dans le Quart et le Cinquième livre, nous le verrons décliner diverses variations, claires et obscures, sur le motif de l'utopie? 2 .

À sa naissance, le géant Pantagruel, fils de la fille du roi des Amaurotes, rompit brutalement la lignée en provoquant la mort de sa mère, comme pour se réapproprier sur de nouvelles bases l'héritage utopien provisoirement échu à son père Gargantua devenu veuf. À la fin du roman, le jeune héros a vaincu les Dipsodes et leur souverain Anarche, qui harcelaient sans cesse le grand-père Amaurote, roi d'Utopie ; il les a soumis, il a renversé Anarche qui sera réduit au rôle de crieur de sauce verte et il règne désormais sur une nouvelle Utopie, la Dipsodie, colonisée par les Amaurotes. Rejeton hyperactif d'un pays imaginé par un philosophe, Thomas More, qui lui-même avait pleine conscience du caractère paradoxal de son invention, Pantagruel, roi des Dipsodes, domine donc l'utopie dans tous ses recoins, toutes ses nuances et tous ses avatars. Car de même qu'il avait donné à la capitale d'Utopia le nom d'Amaurote (ce qui signifie " aux lignes brouillées " ou "ville de l'ombre ", " cité obscure »), il avait affublé du patronyme d'Hythlodée (" qui dit des sottises ») celui qui, revenu du voyage au cours duquel il avait découvert le pays d'Utopia, allait en donner à ses interlocuteurs la description détaillée. S'emparer de l'utopie, dès sa fondation, c'est jouer sur les mots, tourner et retourner les signes, s'inscrire dans une série de livres en postulant le fait qu'inventer, ce n'est jamais, au mieux, que réinventer.

On peut montrer aussi cela à partir de la si célèbre gravure d'Ambrosius Holbein ornant en frontispice l'édition bâloise de l'Utopie qui est déjà, en 1518, la quatrième. L'image (fig. 1) présente, dans le coin inférieur gauche, les deux interlocuteurs dont la conversation donne corps au récit. Raphaël Hythlodée parle avec vigueur, pointant du doigt vers le vaisseau et la carte de l'île qui occupent l'essentiel de la gravure. En face de lui est représenté l'homme qui rapporte le récit d'Hythlodée, le narrateur qu'on a toutes les raisons d'identifier à Thomas More lui-même et que l'on voit écouter, esquissant un geste de sa main gauche et tenant dans sa main droite quelque chose qui ressemble à un livre. Une autre illustration (fig. 2), dans la même édition, montre les interlocuteurs assis dans un jardin, entourés d'un côté par d'élégantes bâtisses et, de l'autre, par les coteaux cultivés d'une campagne avenante ; cité d'un côté, pastorale de l'autre - action et imagination. Là, More que l'on peut identifier cette fois avec certitude puisqu'il est désigné par son nom, tient sur les genoux un objet reconnaissable sans aucun doute : c'est bien un livre. Quel livre ? Un simple indice qui servirait d'emblème à l'humaniste ? L'un de ceux qu'il avait écrits avant l'Utopie? Un recueil de cartes géographiques? Une autre relation de voyage ? La tentation est forte d'y voir plutôt l'Utopie elle-même, récit incarné par Hythlodée, le « diseur de sottises », et qui n'est rien sans dépositaire, ni réceptacle. La première page de la première utopie semble postuler ainsi que l'utopie est un livre, qu'elle est même la série de livres qui se déploie derrière et devant chacun d'eux.

2. À propos de Rabelais et l'utopie, voir notamment Demonet (2012). 
Figure 1. Ambrosius Holbein, illustration en frontispice de l'édition de l'Utopie de Bâle, 1518.
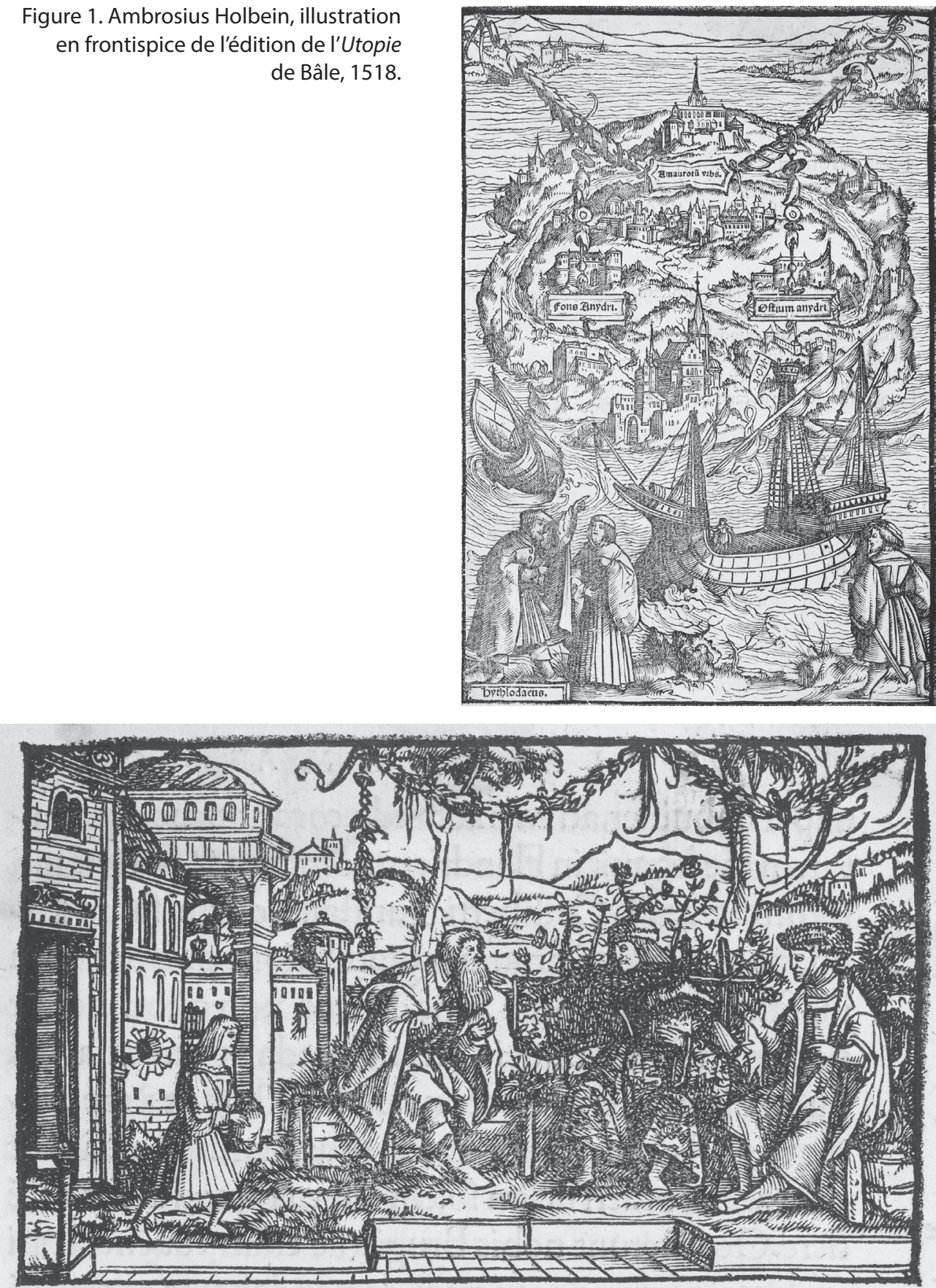

10.Clemens, Hythlodxus, Tho.Morus. Pet, Aegid.

Figure 2. Ambrosius Holbein, illustration pour l'édition de l'Utopie de Bâle, 1518. 


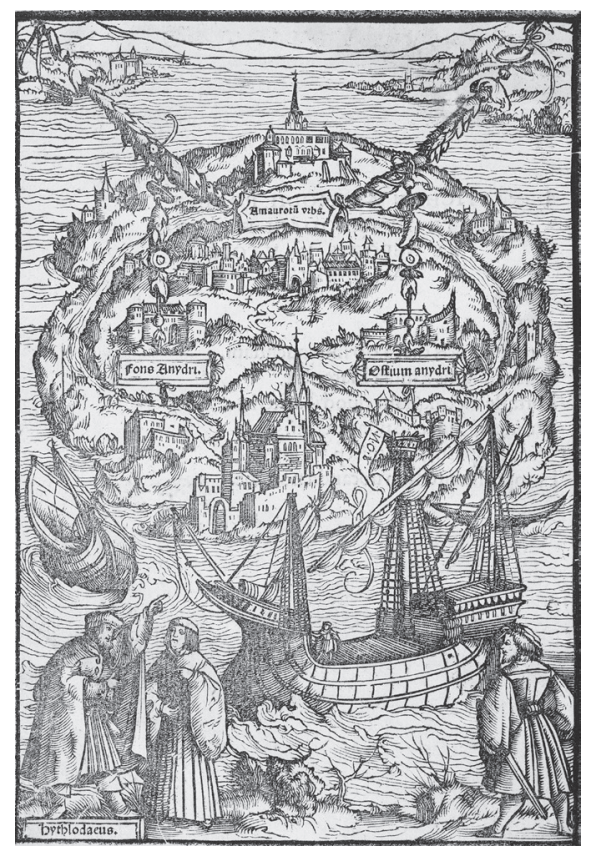

Figure 1. Ambrosius Holbein, illustration en frontispice de l'édition de l'Utopie de Bâle, 1518.

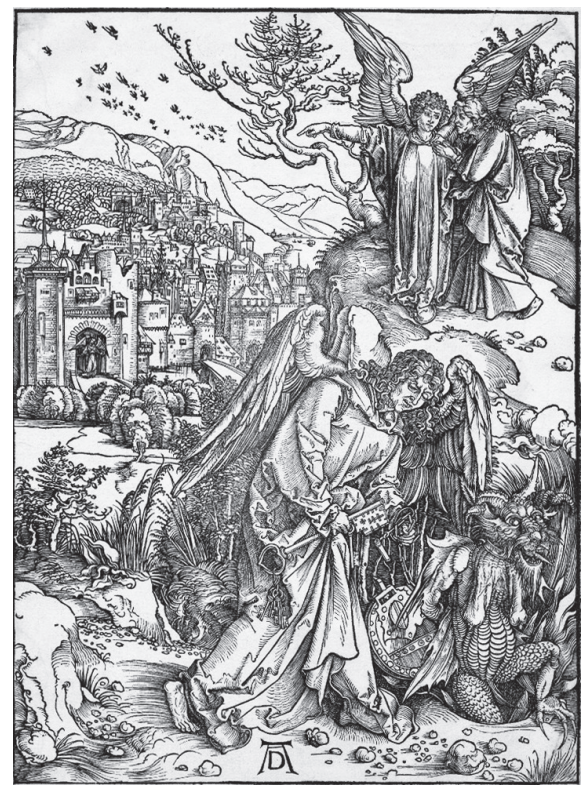

Figure 3. Albrecht Dürer, $15^{\mathrm{e}}$ gravure du cycle de l'Apocalypse, 1496-1498.
Car cette image a beau illustrer une véritable invention (l'utopie), elle n'est elle-même pas vraiment nouvelle dans sa composition. On peut le constater en considérant la succession des gravures qui, depuis le $\mathrm{XV}^{\mathrm{e}}$ siècle, ont servi d'illustration pour la scène de l'Apocalypse où «L'un des sept anges [...] me transporta en esprit sur une grande et haute montagne, et il me montra la cité sainte, Jérusalem, qui descendait du ciel d'auprès de Dieu, brillante de la gloire de Dieu " (La Sainte Bible, 1960, Ap. 21 : 10). Albrecht Dürer (1496-1498 - fig. 3) et Hans Holbein le Jeune (le frère d'Ambrosius 1523 - fig. 4) offrent deux exemples parlants, l'un peu avant, l'autre juste après la réalisation du frontispice de l'Utopie:

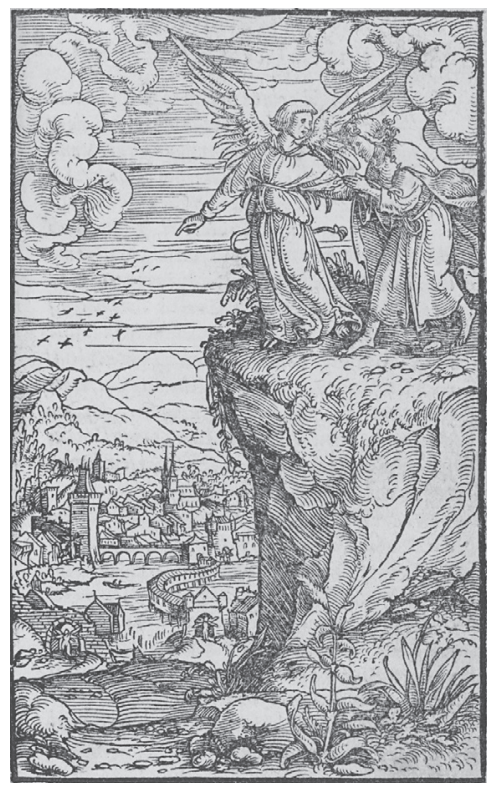

Figure 4. Hans Holbein le Jeune, La Révélation de saint Jean, dans Le Nouveau Testament, Bâle, 1523. 
Entre la représentation de la Jérusalem céleste et celle de l'utopie, il y a eu une inversion sur l'axe de la verticalité, mais le dispositif est resté le même : l'objet est désigné par des observateurs qui sont extérieurs à lui. En outre, les nuées sont devenues un simple ornement, l'ange a cédé sa place à un explorateur bien en chair, saint Jean à un humaniste attentif ; quant aux oiseaux qui assurent le lien entre le lieu d'où l'on regarde et le lieu regardé, ils ont été remplacés par des bateaux. S'il y a claire désacralisation du motif, il y en a tout aussi claire reprise et il en va pareillement avec l'idée même de constituer un monde alternatif qui pourrait être meilleur : Utopie est un nom nouveau qui désigne un héritage ancien, nommé tantôt République, Atlantide, Arcadie, Cité de Dieu, Royaume du Prêtre Jean ou de la reine de Saba, etc. Le nouveau ne serait donc jamais qu'une reconfiguration de l'ancien.

À propos du livre fondateur de Thomas More, il faut rappeler également une chose souvent négligée : il se présente lui-même comme un diptyque. Il y a certes la description détaillée de l'île imaginaire d'Utopia qui donne à voir les caractéristiques d'un monde postulé comme meilleur, mais il y a d'abord la première partie du livre qui présente des opinions critiques sur la réalité politique et sociale de l'Angleterre et de la France. En France, ce sont les ambitions conquérantes d'un roi tout puissant qui sont mises en cause en tant qu'elles génèrent l'essentiel des malheurs publics. En Angleterre, ces mêmes fâcheux effets sont imputés à la cupidité et à l'incurie de la noblesse dans son ensemble qui, en s'appuyant sur des lois injustes, jette dans la misère de trop nombreux citoyens. Ces pays, assurément, devraient être réformés de toute urgence.

Le récit de la découverte de l'île d'Utopia est donc assumé par un personnage ouvertement imaginaire, le « diseur de sottises », ce qui montre bien que More prend soin de présenter son monde inventé comme un univers de pures paroles, sans ancrage référentiel dans la réalité. Mais en même temps, il nous est dit qu'Hythlodée aurait été un compagnon d'expédition de l'authentique navigateur Amerigo Vespucci. Fiction avouée ostensiblement, certes, mais fiction greffée comme une bouture exogène à la réalité des expéditions et des grandes découvertes qui marquent si fortement les temps de More lui-même. Hythlodée aurait abordé en Utopie, mais avant cela, il avait vu la France, l'Angleterre et bien d'autres pays connus. Ainsi l'inventeur de l'utopie postule-t-il que celle-ci est un discours de fiction qui dédouble la description du monde réel, sans toutefois se confondre avec elle. Entre ces deux dimensions, il y a à la fois une distance à ne pas négliger et une intime relation de complémentarité.

Hythlodée, dans le livre, raconte son histoire à Thomas More lui-même, homme d'État et figure intellectuelle majeure de son temps, ainsi qu’à leur hôte Pierre Gilles, autre humaniste de renom. La gravure d'Ambrosius Holbein qui montre les deux personnages échangeant au sujet de l'utopie doit nous faire comprendre qu'un modèle intellectuel projeté, nourri par l'imagination et les désirs (en un mot, une utopie), n'a de véritable lisibilité que dans sa confrontation avec la réalité de ses inventeurs et dans les discours qu'ils tiennent à son sujet. Cette disposition anthropologique 
fondamentale que met en lumière l'utopie telle que More l'a instituée permet aux humains de se voir en même temps comme ils sont et comme ils pourraient être.

Mais ces deux capacités complémentaires ne cohabitent pas sans tension. Thomas More nous en donne encore une frappante confirmation avec la phrase sibylline qui ponctue son Utopia : s'avouant perplexe à l'issue du tableau dressé par Hythlodée de ce monde imaginé, peut-être meilleur, More finit par conclure à son sujet, entre admiration et rejet : « je le souhaite plus que je ne l'espère » (1990, p. 204). Entre aspirer à un monde meilleur et espérer qu'il advienne au milieu de nous, il y a une importante différence qui nous impose de bien faire la distinction entre les capacités d'invention ainsi que de projection de l'esprit humain et les contingences qui président à nos vies dans la réalité physique et sociale qui est la nôtre. L'histoire de l'utopie reposera durablement sur cette irréductible tension entre deux tendances : d'un côté, l'expérience décevante du réel qui inspire, sous l'empire de la littérature et de l'imagination, des visions de modalités alternatives pour le gouvernement, l'organisation et la cohésion des sociétés et, de l'autre côté, des volontés fermes de changer cette trop imparfaite réalité, des velléités plus ou moins virulentes de réforme, voire de renversement radical. Toute utopie invite alors à s'interroger : a-t-elle été écrite pour contribuer à l'enrichissement du réservoir auquel vient puiser l'imagination des humains? Pour dire à ceux-ci qu'un autre monde est possible ? Pour leur proposer un programme de réforme ou même pour les pousser vers une action réformatrice décidée? Gargantua, lui encore, en neutralisant le roi Anarche pour instaurer au pays des Dipsodes l'ordre heureux d'utopie montre cependant que, dans la logique du mundus inversus, toutes les affirmations (puisqu'elles reposent sur des mots) et toutes les croyances que celles-ci génèrent sont susceptibles de retournement : c'est une ancienne anarchie que serait venu supplanter l'ordre nouveau de l'hyperfictif Gargantua. Détrôner les rois ou les remplacer reste ainsi toujours, sous le régime de l'utopie, une opération hypothétique.

\section{Le roi}

De fait les rois, les vrais, ceux qui ne s'appellent pas Anarche, ne pullulent pas dans les utopies sous l'ancien-régime, et pour cause : comment imaginer un pays réformé, conçu sur de nouvelles bases, fondé sur des lois sagement constituées, qui serait dirigé et légitimé par une de ces têtes à couronne qui incarnent justement dans le monde réel des écrivains et des lecteurs la continuité, la permanence de cet ordre d'ancienne souche qui suscite, à la mesure de ses imperfections, des désirs de changement, l'espoir d'un ordre nouveau et inspire ces projections mises en récit que sont les utopies ? Pourtant, les gouvernants s'imposent généralement en utopie par un pouvoir fortement centralisé garanti par des lois contraignantes qui soumettent les individus au collectif, lequel n'est assuré qu'aussi longtemps que le législateur-gouvernant obéit lui-même à cet impératif du bien commun - c'est-à-dire pas très longtemps comme 
viennent déjà le montrer, avant les grandes dystopies des siècles suivants, les intuitions pessimistes de Swift ou de l'abbé Prévost ${ }^{3}$.

Avec la Révolution, arrivent pour les rois des temps difficiles qui se répercutent à plus forte raison dans le patrimoine utopien. Peu d'auteurs le disent avec autant de netteté que l'enthousiaste jacobin Sylvain Maréchal. Au début de 1789, c'est-à-dire, notons-le bien, avant les événements cruciaux de juillet, il publie un recueil intitulé Apologues modernes à l'usage du dauphin; premières leçons du fils aîné d'un roi, comme pour imiter le grand précepteur et sage utopiste Fénelon. L'un des brefs textes qui constituent cette collection s'intitule «Vision. L'isle déserte ». Comme le titre l'indique, c'est un rêve :

un visionnaire se livra au sommeil, \& rêva que tous les peuples de la terre, le jour des saturnales, se donnèrent le mot pour se saisir de la personne de leurs rois, chacun de son côté. Ils convinrent en même temps d'un rendez-vous général, pour rassembler cette poignée d'individus couronnés, \& de [sic] les reléguer dans une petite isle inhabitée, mais habitable ; le sol fertile n’attendoit que des bras \& une légère culture. (Maréchal, 1789, p. 30-31)

La fin est celle que l'on pouvait attendre : ces rois dégradés (comme Anarche devenu crieur de sauce verte), livrés à eux-mêmes, dépossédés des signes et de la réalité du pouvoir, contraints de partager leur sort avec leurs semblables pareillement déchus " ne vécu[ren]t pas longtemps en paix ; \& le genre humain, spectateur tranquille, eut la satisfaction de se voir délivré de ses tyrans par leurs propres mains » (Maréchal, 1789, p. 31).

Quatre ans plus tard, Maréchal se ressaisit de ce motif pour en faire, cette fois-ci, une pièce de théâtre à faire jouer devant le peuple de Paris au plus fort de l'effervescence révolutionnaire de 1793 : Le Jugement dernier des rois. La pièce sera effectivement montée "sur le Théâtre de la République, au mois Vendémiaire et jours suivants » de "l'an second de la République française, une et indivisible », précisément le 17 octobre, soit au lendemain de l'exécution de Marie-Antoinette et au moment même où le calendrier révolutionnaire entrait en vigueur (6 octobre 1793) ${ }^{4}$. L'auteur ne rêve plus, il imagine seulement ou mieux même, il prévoit (désignant génériquement sa pièce comme une " prophétie en un acte, en prose $»^{5}$ ), que toute l'Europe est devenue républicaine et qu'en vertu du jugement prononcé contre les rois par une as-

3. On renvoie ici bien sûr aux Voyages de Gulliver (1721) et à Le Philosophe anglais, ou Histoire de M. Cleveland, fils naturel de Cromwell (1731-39) ; à propos du modèle utopique dans le roman de l'abbé Prévost, voir Rosset (2010). Plus généralement, voir au moins Racault (1991).

4. À propos de cette pièce, voir en particulier Matyaszewski (2020). Outre une fine analyse de la pièce, on y trouve un recensement précis des études consacrées à Maréchal et au Jugement dernier des rois.

5. Cette précision, ainsi que les deux précédentes figurent sur la page de titre de l'édition originale de la pièce «A Paris, de l'imp. de C.-F. Patris, imprimeur de la Com. Rue du fauxbourg St.-Jacques, aux ci-devant Dames Ste.-Marie », 1793. 
semblée de sans-culottes de tous pays, les souverains de son temps (pape compris) sont exilés sur une île déserte, condamnés à s'entre-déchirer avant de disparaitre dans l'éruption apocalyptique d'un volcan. Les sans-culottes, unis fraternellement aux sauvages des îles avoisinantes, savourent le spectacle à distance comme un feu d'artifice libérateur.

Il y a peu, l'île déserte était ce lieu propre à abriter les fantasmes politiques des mécontents, des rêveurs ou des utopistes ; le rêve des réformateurs ayant pris forme dans la réalité avec l'assaut de la Bastille et ses suites, elle est désormais vouée à accueillir les ci-devant maitres, soit le rebut de cette humanité qui vient dêtre restituée dans sa dignité. L'utopie, voulait-on croire, s'était alors installée sur le continent, sur la terre ferme du réel. Un peu naïvement, certes, mais avec la conviction euphorique du citoyen de 1793, Maréchal figure le rapatriement sur le continent, dans les terres habitées par les hommes réels, des projections et des expériences qu'ils avaient dû confiner jusqu'ici dans des lointains insulaires. L'utopie n'est plus rêvée dans un ailleurs prudemment circonscrit, elle est réalisée au milieu de nous; elle ne serait donc plus utopie dans cette nouvelle réalité où les législateurs censément vertueux ont remplacé les rois. Les rois de Maréchal sont bel et bien périmés, ils sont comme le reliquat d'un temps où un monde sans eux ne pouvait être que fantasmé.

De là, la thèse selon laquelle l'utopie aurait été, voire serait encore l'incubateur des révolutions, l'espace où s'expérimentent mentalement et discursivement des modèles alternatifs qu'il s'agira un jour de mettre en pratique, semble s'imposer de toute évidence, et il est tentant de l'embrasser. Or l'exemple d'un roi authentique, d'un roi polonais, vient pour ainsi dire rappeler la sentence finale de Thomas More qui disait souhaiter l'utopie plus qu'il ne l'espérait, laissant entendre par-là - assez énigmatiquement il est vrai - qu'il vaudrait mieux ne pas confondre les désirs et leur accomplissement, l'imagination et l'action, la fiction et la réalité.

\section{L'utopie d'un roi polonais}

À l'expression « roi polonais », au XVIII siècle, on pouvait pratiquement appliquer l'observation que Louis-Sébastien Mercier avait un jour formulée pour commenter le syntagme "poète suisse » : "l'accouplement seul de ces deux mots fait rire, n'estil pas vrai, lecteur ?» (1784, p. 39). Depuis longtemps, le statut de ce roi élu par la diète des nobles et limité dans son pouvoir par un contrat (les pacta conventa), suscitait l'étonnement en Europe. Entre curiosité et raillerie, les bons mots s'étaient multipliés : «il semble que le roi n'en soit souverain que sous bénéfice d'inventaire » (Camus, 1625, t. I, p. 2) ou encore : « le roi de Pologne est comme celui des mouches à miel. Il n'a point d'aiguillon et ne peut faire de mal à ses sujets " (Le Laboureur, 1647, p. 10), alors que dans son uchronie L'An 2440 (1770), le même Louis-Sébastien Mercier voyait le royaume de Pologne enfin revenu à une situation de paix et de prospérité grâce au retour à une couronne héréditaire, grâce à l'effacement d'une anoma- 
lie $^{6}$. Il fallait donc fort paradoxalement l'imagination d'un utopiste pour remettre à sa juste place monarchique ce souverain-marionnette exposé à toutes les tempêtes soufflant de l'intérieur comme de l'extérieur. Pour autant, bien sûr, que soit postulée (comme dans le roman de Mercier), la pérennité bienfaisante du système monarchique.

Parmi tous ses confrères, c'est sans doute à Stanislas Leszczyński que revient le titre du plus romanesque des rois de Pologne. Élu au trône de son pays à deux reprises, mais écarté chaque fois par les manœuvres conjuguées des factions internes et des souverains voisins, il était devenu un roi idéal qui n'avait pas eu à compromettre son titre par l'exercice effectif du pouvoir. Débonnaire, généreux, devenu de surcroît beau-père de Louis XV et souverain du paisible duché de Lorraine et de Bar, il réunissait toutes les qualités qu'il fallait conjuguer pour incarner la figure du bon roi. D'où le nombre important de poèmes, de chansons, de drames, d'anecdotes, de nouvelles et de romans qui lui furent consacrés. Tel ce roman de $\mathrm{M}^{\mathrm{me}}$ de Renneville, paru en 1787, où l'on peut lire ces lignes, dans un préambule adressé au lecteur :

Pour rendre un héros intéressant, on a souvent recours à la fiction ; on peint l'homme comme on voudrait qu'il fût, et non tel qu'il est ; ici l'imagination n'a rien à inventer. Stanislas, favorisé par la nature, doué des avantages de la naissance et des qualités les plus propres à supporter les revers de la fortune, Stanislas excite l'admiration et l'attendrissement ; le cœur conduit la plume en écrivant cette histoire : c'est celle d'un roi bienfaisant. Que serviraient les ornements de l'esprit ? La vertu sans art est si belle. ( $\mathrm{M}^{\mathrm{me}}$ de Renneville, 1807 , t. I, p. 9)

C'est donc une de ces histoires de rois qu'on aime à lire, tant celles qui nous viennent ordinairement de l'expérience sont décevantes. Et le fait que ce roman a été édité d'abord en 1787, puis une deuxième fois en 1807 et une troisième en 1818 montre bien qu'indépendamment des vicissitudes politiques du tournant du siècle, le besoin de telles représentations restait constant.

Leszczyński ne s'est toutefois pas contenté de vivre tranquillement sa vie de souverain sans périls et de s'incarner ainsi, sans le savoir, en possible héros d'utopie, puisqu'il s'est fait lui-même l'auteur d'un petit roman utopique : L'Entretien d'un Européen avec un insulaire du royaume de Dumocala, qui n'est certainement pas le texte le plus admirable que le XVIII ${ }^{e}$ siècle aura produit en matière d'utopie, sans qu'il soit toutefois dénué d'intérêt ${ }^{7}$. Il parut en 1752, avec une nouvelle édition en 1754 (reprise l'année suivante) « à laquelle on a joint les Extraits \& les Jugemens

6. À propos des figures du roi de Pologne dans les fictions françaises, voir Rosset (1996), p. 97-133.

7. Voir Leszczynski (1981). Le texte intégral est aussi repris dans l'anthologie préparée par A. Muratori-Philip, Stanislas Leszczynski, aventurier, philosophe et mécène des Lumières (2005), p. 929952. Pour des aperçus détaillés et savants sur cette utopie de Leszczyński, voir l'introduction de L. Versini à son édition (Leszczynski, 1981) ainsi que Versini (2010). 
qui ont paru dans quelques Journaux ", avant d'être inséré dans la collection en quatre volumes des Euvres du Philosophe bienfaisant (1763).

Il semblerait à première vue difficile d'assortir au portrait-type des auteurs d'utopie, héritiers de la tradition libertine du Grand Siècle et souvent tenus pour responsables du grand tournant révolutionnaire, ce roi très fier de tous ses titres - quand bien même ils n'étaient justement que des titres - , ce serviteur convaincu de la foi et de l'église catholiques, ce conservateur bon teint. Impossible pourtant de dénier au bon roi Stanislas sa contribution à la tradition littéraire de l'utopie, mais peut-être aussi, indirectement, à une vision plus nuancée de cette pratique littéraire si évidemment politique. Il nous permet en tout cas de rappeler qu'au siècle des Lumières, l'utopie s'est imposée comme un modèle de récit et de discours, non plus forcément comme un véhicule d’opinion. Mais des plus timides aux plus virulentes, toutes ses actualisations sont l'expression de cette énergie si particulière qui inspire dans tous les milieux de ce temps des perspectives de réforme et plus encore : une conviction que l'homme est capable d'en concrétiser au moins un certain nombre, dans un certain périmètre. C'est sur ce nombre et sur l'extension du périmètre que diffèrent entre eux les auteurs des quelques 150 textes utopiques produits au XVIII ${ }^{e}$ siècle en français ou traduits dans cette langue ${ }^{8}$.

Jamais les utopies ne furent si nombreuses et jamais non plus si variées, si riches et si surprenantes dans leurs différences mêmes. Limiter l'utopie aux textes qui pourraient faire office d'annonciateurs, voire d'inspirateurs de la Révolution est un profond malentendu. Péché mortel de téléologie, bien sûr, mais avant tout délit d'ignorance. Il faut donner à connaître ce corpus de textes si ample et diversifié pour permettre de mesurer tout ce qui s'y révèle : la puissance du désir de mieux vivre, l'étendue de l'inventivité des hommes, le foisonnement de leur imagination. Tous, ils semblent raconter la même histoire sur un canevas peu propice aux profondes variations ; mais chacun d'eux donne à voir, à rêver, à postuler d'autres hommes, d'autres coutumes, d'autres principes, d'autres solutions et à interpréter d'autres figures, d'autres scènes, d'autres séquences. Et tous font cela parce qu'ils perçoivent la réalité sociale dans laquelle ils vivent comme insatisfaisante ou complètement insupportable.

En tant que roi doublement déchu et doublement victime, Stanislas Leszczyński avait de bonnes raisons d'estimer que la monarchie elle-même (qu'il ne s'agissait nullement pour lui de mettre en cause en tant que telle) ainsi que le réseau agité des différentes monarchies de l'Europe pouvaient être améliorés. D’où la leçon livrée dans son petit roman et exposée encore plus explicitement dans un texte associé à ce dernier dès la deuxième édition, Réponse à la lettre d'un ami, où sa majesté l'auteur confirme clairement qu'il n'avait pas voulu simplement s'amuser en divaguant de façon romanesque, mais donner surtout quelques idées applicables :

8. Voir notamment Baczko, Porret et Rosset (2016). 
Vous ne l'envisagez peut-être que comme l'amusement d'un philosophe, ou comme la production d'un génie oisif, tout au plus comme un de ces romans politiques dont Platon a donné l'idée.

Pour moi, je n’en pense pas tout à fait de même, j’aperçois d'utiles vérités sous un récit qui paraît fabuleux ; et il me prend envie de réaliser ici ce qui vous semble chimérique. (Leszczyński, 2005, p. 953)

De fait, aucun autre auteur d'utopie n'avait eu réellement les moyens de dire et de faire ainsi, puisque l'ici dont parle Stanislas, ce n'est pas, généralement, l'ici-bas des humains, mais le duché prospère qu'il dirigeait avec une autonomie suffisante pour pouvoir y exercer sagement le pouvoir à l'image du souverain de Dumocala. Cela dit, on sait aussi que le beau-père du roi de France avait tout autant à l'esprit son propre pays, la Pologne, qui nécessitait de profondes réformes, comme les décennies suivantes allaient le prouver dramatiquement. Son roman, ainsi que le seront plus tard Les lois de Minos de Voltaire (1773) ou Le voyage de Hafez de Jean Potocki (1792), peut être lu comme une réflexion déguisée sur la situation polonaise ; il anticipe également ces fameux textes de Rousseau (Considérations sur le gouvernement de Pologne - écrit en 1770-71) et de Mably (Du gouvernement et des lois de la Pologne - écrit également en 1770-71) qui furent souvent considérés comme des romans politiques, voire des utopies?.

Dumocala, comme Utopia, est un nom parlant. En polonais, le verbe dumać signifie songer, rêver. C'est un pays ouvertement conjecturé dont les vertus sont décrites par un vieux sage à un voyageur européen égaré. Ce pays possède évidemment toutes les qualités attendues; il est donc tout le contraire de l'Europe, dont le vieux sage dit connaître les vices pour avoir eu l'occasion de lire un traité français de l'histoire universelle (celui de Bossuet ?). Les deux interlocuteurs représentent ainsi l'opposition souvent constituée dans l'orbe de l'utopie entre l'historien qui, d'après les mots de Charles-Georges-Thomas Garnier, « peint les hommes tels qu'ils ont été ou tels qu'ils sont » et le philosophe qui, toujours selon Garnier, "se transporte dans des mondes nouveaux, où il recueille des observations qui ne sont ni moins intéressantes, ni moins précieuses ${ }^{10}$. L'utopie, domaine par excellence du philosophe s'avère ainsi très vaste au temps où toute réflexion critique dans tout canton de la pensée et du savoir est précisément qualifiée de philosophique. De là à soutenir qu'utopie et Lumières ont tendance à s'unir dans un rapport d'emblématisation des unes par l'autre, il n'y a qu'un pas que Leszczyński et beaucoup de ses comparses en utopographie nous invitent à franchir.

9. Voir par exemple Melchior Grimm qui qualifiait le Rousseau auteur des Considérations sur le gouvernement de Pologne de "philosophe oisif qui emploie son loisir à esquisser des lois et une forme de gouvernement pour quelque utopie ». Dans Correspondance inédite de Grimm et de Diderot (1829, p. 343).

10. C.-G.-T. Garnier est l'éditeur de la célèbre série des Voyages imaginaires, songes, visions et romans cabalistiques publiée à Paris en 36 volumes de 1787 à 1789. Pour les passages cités, voir t. I, p. 1 . 
Critiquer le monde pour en conjecturer, en postuler un autre - en d'autres termes, philosopher - est une posture qui peut autant conduire à l'action radicale qu'à la prudente retenue. On ne sera pas étonné d'apprendre que c'est cette deuxième attitude qu'incarne le roi Stanislas. Son petit roman est encadré par deux phrases qui disent la même chose : « qu'il est triste que le bonheur humain ne se rencontre qu'en des pays inconnus qui nous sont inaccessibles " lisons-nous dans l'Avertissement ; quant à la dernière phrase du texte, elle résonne ainsi : " mon lecteur [...] pourra juger que le vrai bonheur des peuples ne se trouve que dans les régions inconnues " (Leszczyński, 1981, p. 1 et 45). Position résignée qui, sous un angle politique, peut être perçue comme une dérobade devant les désirs et les projets de changement dans le monde imparfait des hommes, mais qui, d'un point de vue littéraire, redit quelque chose de capital : la force de la fiction, qui n'est pas seulement, comme le voudrait ce roi presque fantoche, un refuge possible et suffisant pour les hommes en quête de bonheur, mais un champ d'incubation et d'expérimentation pour les idées ou encore, plus essentiellement, le lieu où l'homme s'est érigé le théâtre de sa vie intérieure. Et qui saurait contester la réalité de celle-ci ? la réalité véhémente, par exemple, du désir de bonheur ? La Révolution ne fut pas l'aboutissement de l'utopie, ni la conséquence de ce désir, mais elle apporta la preuve de la réalité de ce désir, la preuve que les mondes inconnus sont partie intégrante du monde que l'on croit connaître. C'est pourquoi bien des utopies peuvent être lues comme des fables vouées à l'explication, à la justification et à l'illustration de la fiction même.

Stanislas Leszczyński n’avait fort probablement pas conscience de cette dimension réflexive de l'utopie comme genre littéraire. Son roman comporte seulement une série d'observations et de propositions qui pouvaient être, selon lui, appliquées par un souverain sage et vertueux. Pour dire cela, il s'est pour ainsi dire inscrit dans la généalogie des utopistes, tel un descendant de More et de Rabelais (ou de Pantagruel, leur représentant fictif), en enrichissant modestement d'un petit volume la grande bibliothèque des utopies. Mais il y a, dans son geste, quelque chose de fort extraordinaire. Car en tant qu'auteur de ce texte et souverain appelé à agir dans son duché de Lorraine et de Bar, il se figurait en somme lui-même en héros d'utopie. Il présente en ce sens un cas unique où le rapport entre réalité et fiction utopique se noue dans cette seule et même personne qu'est l'auteur du texte, le souverain qui y est figuré et celui qui est appelé à gouverner selon le modèle promu par la fiction. Le roi de l'utopie, c'est lui. Ainsi, n'est-ce pas sans justesse que Fréron, qui connaissait bien l'identité de l'auteur, termine son compte rendu du roman sur ces mots : "Si c'étoit un particulier qui eût fait cet ouvrage, il seroit digne que les Rois le prissent pour leur Ministre. Si c'étoit un Souverain, il mériteroit d’être placé sur le trône de l'Univers » (Leszczyński, 1755, p. 170). 


\section{RÉFÉRENCES}

Baczko, B., Porret, M. et Rosset, F. (dir.). (2016). Dictionnaire critique de l'utopie au temps des Lumières. Genève : Georg.

Camus, J.-P. (1625). L'Iphigène de Mr. De Belley. Rigueur sarmatique. T. I. Lyon : chez Antoine Chard.

Correspondance inédite de Grimm et de Diderot. (1829). Paris : H. Fournier.

Demonet, M.-L. (2012). Utopie et dystopie chez Rabelais, de Pantagruel au Quart Livre. Revista MORUS. Utopia e Renascimento. "Utopie, consensus et libre arbitre (XIV ${ }^{\mathrm{e}}-\mathrm{XVII}^{\mathrm{e}}$ siècles) : Fais ce que voudras ». C. Berriel, M.-L. Demonet (éds.), 8, 105-118.

http://www.revistamorus.com.br/index.php/morus/article/view/118

La Sainte Bible. (1960). (Chanoine A. Crampon trad.). Paris, Tournai, Rome, New York: Desclée \& Cie Éditeurs.

Le Laboureur, J. (1647). Traité particulier du Royaume de Pologne. Paris : La Veuve J. Camusat, et P. Le Petit.

Leszczyński, S. (1981) [1752]. Entretien d’un Européen avec un insulaire du royaume de Dumocala. L. Versini (éd.). Nancy : Publications de l’Université de Nancy.

Leszczyński, S. (1755). Entretien d'un Européan avec un insulaire du Royaume de Dumocala. Paris : Chez Duchesne.

Leszczyński, S. (2005) [1754]. Réponse à la lettre d’un ami. Dans A. Muratori-Philip (éd.), Stanislas Leszczynski, aventurier, philosophe et mécène des Lumières (p. 952-967). Paris : Laffont.

Mably, G. Bonnot de. (2008) [1770-1771]. Du gouvernement et des lois de la Pologne. M. Belissa (éd.). Paris : Kimé.

Maréchal, S. (1789). Apologues modernes à l'usage du dauphin; premières leçons du fils aîné d'un roi. Bruxelles : s.n.

Maréchal, S. (1793). Le Jugement dernier des rois. Paris : l'Imprimerie de C.-F. Patris, l'an second de la République Française, une et indivisible.

Matyaszewski, P. (2020). La possibilité d'une île, ou Le Jugement dernier des rois de Sylvain Maréchal (1793). Cahiers ERTA, 22, 71-88. https://doi.org/10.4467/23538953CE.20.009. 12427

Mercier, L.-S. (1770). L'An 2440. Rêve s'il en fut jamais. Amsterdam : Van Harrevelt.

Mercier, L.-S. (1784). Mon bonnet de nuit. Neuchâtel : Société Typographique.

More, T. (1516). Libellus vere aureus, nec minus salutaris quam festivus, de optimo reipublicae statu deque nova insula Utopia. Louvain : Thierry Martens.

More, T. (1990). L'Utopie. Dans Voyages aux pays de nulle part. F. Lacassin (éd.). Paris : Laffont.

Rabelais, F. (1973). Euvres complètes. G. Demerson (éd.). Paris : Éditions du Seuil.

Racault, J.-M. (1991). L'Utopie narrative en France et en Angleterre 1675-1751. Oxford : The Voltaire Foundation.

Renneville, $\mathrm{M}^{\mathrm{me}}$ de. (1807) [1787]. Stanislas, roi de Pologne. T. I. Paris : Ch. Villet.

Rosset, F. (1996). L'Arbre de Cracovie. Le mythe polonais dans la littérature française. Paris: Imago.

Rosset, F. (2010). La solution utopique dans Cleveland. Dans L. Fraisse (dir.), Séries et variations. Études littéraires offertes à Sylvain Menant (p. 137-148). Paris : PUPS.

Rousseau, J.-J. (1964) [1770-1771]. Considérations sur le gouvernement de Pologne. Dans Euvres complètes. T. III. J. Fabre (éd.). Paris : Gallimard. 
Versini, L. (2010). Une utopie réalisable et en partie réalisée : le programme de La Voix libre du citoyen et de Dumocala. Dans A. Hatzenberger (éd.), Utopies des Lumières (p. 53-76). Lyon : ENS Éditions.

Voyages imaginaires, songes, visions et romans cabalistiques. (1787). T. 1. C.-G.-T. Garnier (éd.). Amsterdam, Paris : s.n.

Crédits d'illustrations

Fig. 1-3 - Wikimedia under the Creative Commons Attribution-Share Alike 4.0 International licence. Fig. 4 - (C) The Trustees of the British Museum. Shared under a Creative Commons Attribution-NonCommercial-ShareAlike 4.0 International (CC BY-NC-SA 4.0) licence.

RÉSUMÉ : Après avoir rappelé la figure de Pantagruel, petit-fils du roi d'Utopie et roi des Dipsodes, qui permet d'évoquer quelques propriétés de l'utopie (et en particulier celle qui consiste à figurer, à l'intérieur d'elle-même, les liens de filiation qu'elle entretient avec des textes antécédents), le propos se resserre sur la figure du roi dans la tradition de l'utopie, notamment avec l'exemple du Jugement dernier des rois de Sylvain Maréchal. L'attention est ensuite plus spécifiquement dirigée vers la figure du roi de Pologne, particulièrement propre à stimuler l'imaginaire politique des Européens au XVIII ${ }^{e}$ siècle, puis sur la personne de Stanislas Leszczyński, seul souverain auteur d'utopie, avec son petit roman Entretien d'un Européan avec un insulaire du Royaume de Dumocala qui est à la fois fidèle au modèle narratif qu'il contribue à perpétuer et dénué d'intentions révolutionnaires.

Mots-clés : Utopie, roi, Pologne, François Rabelais, Sylvain Maréchal, Stanislas Leszczyński

\section{From Amaurote to Dumocala: variations on kings in utopias}

ABSTRACT: After recalling the figure of Pantagruel, a grandson of the king of Utopia and king of the Dipsodes - which makes it possible to evoke some of the properties of utopia, and in particular that which consists of searching within the text for filial links it maintains with previous texts - the discussion is narrowed down to the figure of the king in the tradition of utopia, with Sylvain Maréchal's Last Judgement of Kings serving as an example. Next, attention is directed more specifically to the figure of the king of Poland, a figure particularly suited to stimulate the political imagination of Europeans in the 18th century, and then to the person of Stanislas Leszczyński - the only sovereign to have written a utopia. He is the author of the short novel Entretien d'un Européan avec un insulaire du Royaume de Dumocala, which is both faithful to the narrative model it helps to perpetuate and devoid of any revolutionary intentions.

Keywords: Utopia, king, Poland, François Rabelais, Sylvain Maréchal, Stanislas Leszczyński 